\title{
ANÁLISIS LINGÜÍSTICO DE UNA CARTA DEL SIGLO XVII, CONTACTO MAYA-ESPAÑOL
}

\author{
LINGUISTIC ANALYSIS \\ OF A $17^{\text {th }}$ CENTURY LETTER, \\ CONTACT MAYA-SPANISH
}


Esta contribución está centrada en analizar una carta del siglo XVII escrita por una mujer indígena maya. En primer lugar, se trata de estudiar el discurso tomando en cuenta los niveles gráfico, fonológico, morfosintáctico, léxico y semántico. Nuestro objetivo consiste en advertir que fenómenos tales como: la falta de concordancia, la falta de uso de conectores en el discurso, entre otros, se deben a la influencia de la lengua materna.

Palabras Clave: análisis filológico, análisis lingüístico, lenguas en contacto

This contribution is focused on analyzing the seventeenth century of an letter written by an indigenous Maya woman. First, it is analyzing the speech taking into account the levels phonological, morphosyntactic, lexical and semantic. Our goal is to see that these phenomena: no concordance, the lack of connectors in speech, among others, due to the influence of the mother tongue.

KEY WORDS: philological analysis, linguistics analysis, language in contact 


\section{ANÁLISIS LINGÜÍSTICO \\ DE UNA CARTA DEL SIGLO XVII, CONTACTO MAYA-ESPAÑOL}

\section{LINGUISTIC ANALYSIS OF A $17^{\text {th }}$ CENTURY LETTER, CONTACT MAYA-SPANISH}

Idanely Mora Peralta Universidad Nacional Autónoma de México

Afortunadamente aún contamos con documentos invaluables que permiten a los investigadores de diferentes disciplinas conocer y reconstruir nuestro legado histórico y lingüístico; nos referimos a los manuscritos que se resguardan en los diferentes archivos. Cada uno conserva entre sus líneas los diferentes saberes de los habitantes de civilizaciones antiguas. Al mismo tiempo, el acercamiento a estos documentos nos permite analizar ciertos fenómenos lingüísticos que se originaron a partir del contacto del español con otras lenguas indígenas.

El documento que ocupa estas páginas forma parte del Corpus Electrónico del Español Colonial Mexicano (COREECOM) y es parte del proyecto Conacyt-CB 2012/180245. Nuestro objetivo es hacer una descripción desde los niveles gráfico, fonológico, morfosintáctico, léxico y semántico, pues consideramos que este estudio puede contribuir al entendimiento de la influencia de las lenguas indígenas en la cons- 
trucción del español del siglo XVII. Nuestra hipótesis se centra en que los fenómenos registrados en este documento se deben a la influencia de la lengua materna: el maya, yucateco.

Para hacerlo accesible se optó por retomar los parámetros que el Grupo de Estudio del Español Colonial Mexicano (GEECOM) ha establecido y que aparecen publicados en el libro de Beatriz Arias Álvarez (2014). Consideramos pertinente esta clasificación archivística porque, contrario a otros sistemas empleados en manuales, no se deja de lado ningún dato del escribiente, ni el tipo de género discursivo. La grafemática es otro aspecto importante en nuestro análisis, ya que se respeta el uso gráfico, la separación y unión de palabras y no se recupera ninguna grafía faltante en la versión paleográfica.

En primer lugar, debemos contar con una ficha en la que se condensen los datos más importantes del manuscrito. Esta fase se compone de dos etapas. La primera se denomina datos del encabezado, cuyo contenido es el siguiente:

Nombre del archivo: Archivo General de la Nación, México. Grupo documental: Inquisición 61, volumen: 281, expediente. 65 , fojas: $858-864$, este documento corresponde al folio: 859 .

Zona geográfica: Nueva España, Península de Yucatán.

Origen étnico-social: indígena.

Sexo del escribiente: mujer.

Tipo de documento: semiformal.

Variedad textual: denuncia.

Periodo: 1600-1620.

Fecha: 1605. 
Destinatario: Su merced, el Lic. Francisco Pacheco, presbítero.

Estos datos nos permiten enmarcar al documento en una región, periodo, tipo de escrito y características del escribiente. En nuestro caso es un documento del siglo XVII, de la zona de la península de Yucatán (maya), es una denuncia y el tipo de registro es semiformal por la carga emotiva que implica la denuncia y por tratarse de una escribiente indígena.

Mientras que la segunda etapa consiste en saber el estado en el que se encuentra el documento, es decir, si está roto o apolillado, ya que esto puede modificar su contenido, asimismo también resulta relevante señalar de qué trata el manuscrito. El documento que nos ocupa está en buen estado y es la denuncia de Clara Ve, mujer indígena, que fue solicitada en confesión. Finalmente, se coloca el nombre de la persona que realizó la recopilación y la edición, en este caso corresponde a quien esto escribe.

En cuanto a la presentación del documento, es necesario que cada manuscrito cuente con dos paleografías: una estrecha y la otra crítica. La primera consiste en que, una vez numerados los renglones, se conservan todas las características del documento desde el tipo de letra hasta la unión de palabras, esto con la finalidad de que "el investigador realice estudios gráfico-fonológicos, morfológicos, de historia de la escritura, así como de evolución de las tradiciones gráficas" (Arias Álvarez, 2014: 31). La segunda es una versión modernizada en la que se incorporan elementos de puntuación, pues se pretende que el lector de cualquier disciplina, que tenga interés en estos manuscritos, obtenga una lectura e interpretación accesibles. 
A continuación presentamos las dos versiones paleográficas de transcripción con los criterios arriba descritos de la carta de Clara Ve. Posteriormente, se procederá con el análisis.

\section{PRESENTACIÓN PALEOGRÁFICA}

1. Eltes timonio falso queme leuãtã por mi suegro fran cen caçiq' ygobernador

2. Enel pueblo de yax cucul Es por un odio quetiene El pe dio de camara comigo

3. por, no aver con çedido conel Enla quares ma pasado fuy acon fesarme conel

4. loprime quehizo con migo Requerdose dixo que yo En tra En la ja

5. cristiya conel a hazer pecado conel yRespondi q' en ninguna manera

6. podia hazer En presençia dejen tesymas Enla yglesi y Joy casad q'

7. veniendo aoydos de mi marido me mal tratara ymedixo buen Remed

8. queyo hazeos En ferma ymuy mala fatigando En buestra casa y en

9. bia buestro marida allamar para confesaros yenton ces cumplire mi vo 
10. luntad En Vos es to medixo yleRespondi pe dad me de confesar y cõ

11. Suela mialma qno vengo eso mas medixo q haria Recojer limosnaen dinero

12. y guapil ynaguas Enlosdias de pasquas yen la fiesta del pueblo q' todo lo que

13. mediere todo os dara y uro marido Esunpobre indio no tiene de q' sus

14. ten tarte q' yo te sus tentare muy bien y mas que yo amare? tumarido y tu

15. Suegro El caçique y Jino vos vro marido y tu Suegro hare pordonde eschar

16. os destepueblo y quitarle $\int$ ucacicazgo y les pondicomo podi hazer En

17. presen çia de mi marido y medixo bus care por donde En biar fuera y ha

18. re mi volunta ami espacio Estos y otras mucha cos me dixo q'aqui,,no tray

19. go es crita En mi cõfision de clarare de pie acabeza

20. hasta agora nome Ecõfesado por no aver hallado pe con quien

21. porque medixo q' no ade abfoluer aunq' yo me co Fies con otro

22. y por Esto En tien que tiene odio comigo y comi $\int u e g r o$ buscando

23. ter çeras personaspara Ser testigos falsos contra mi Su egro

24. y contrami hazien me Este grabio y molestia Eynjuta

25. juro aesta $\dagger$ dios estestigo desta verdad digo Esto por alcan 
26. zar justa y sin malicia ninguna [rubrica]

27. clara ve muger de

28. diego cen

\section{PRESENTACIÓN CRÍTICA}

\section{$\dagger$}

$\{1\}$ El testimonio falso que me levantan por mi suegro Francisco Cen, cacique y gobernador $\{2\}$ en el pueblo de Yaxcucul, es por un odio que tiene el padre Diego de Cámara comigo, $\{3\}$ por no haber concedido con él. En la cuaresma pasado fui a confesarme con él, $\{4\}$ lo primero que hizo conmigo, recuérdose dixo que yo entra en la ja- $\{5\}$ cristiya con él a hacer pecado con él, y respondí que en ninguna manera $\{6\}$ podía hazer en presencia de jentes y más en la iglesia, y soy casada, que $\{7\}$ veniendo a oídos de mi marido me maltratara, y me dixo: 'buen remed $<$ io $>\{8\}$ queyo hazeos enferma y muy mala fatigando en vuestra casa y en- $\{9\}$ bía vuestro marida a llamar para confesaros y entonces cumpliré vo$\{10\}$ luntad'. En vos esto me dixo y le respondí: 'padre dadme de confesar y con- $\{11\}$ suela mi alma que no vengo eso'; mas me dixo que haría recoger limosna en dinero $\{12\} \mathrm{y}$ guapil y naguas en los días de pascuas y en la fiesta del pueblo, que todo lo que $\{13\}$ me diere, todo os dará. Y vuestro marido es un pobre indio no tiene de qué sus-\{14\}tentarte muy bien y más que yo amare? Tu marido y tu\{15\} suegro, el cacique, y si no vas vuestro marido y tu suegro haré por donde eschar-\{16\}os deste pueblo y quitarle su cacicazgo. Y respondí cómo podía hazer en $\{17\}$ presencia de mi marido, 
y me dixo buscaré por dónde enviar fuera y ha- $\{18\}$ ré mi volunta a mi espacio. Estos y otras mucha $\cos <$ as $>$ me dixo que aquí no trai-\{19\} go escrita en mi confisión declararé de pie a cabeza $\{20\}$ hasta agora no me e confesado por no aver hallado $\mathrm{p}<$ adre $>$ con quién, $\{21\}$ porque me dixo que no me a de absolver aunque yo me confies $<\mathrm{e}>$ con otro, $\{22\}$ y por esto en tien $<$ do $>$ que tiene odio $\mathrm{co}<\mathrm{n}>\operatorname{migo}$ y $\mathrm{co}<\mathrm{n}>$ mi suegro, buscando $\{23\}$ terceras personas para ser testigos falsos contra mi suegro $\{24\}$ y contra mí, hazien $<$ do $>$ me este $<$ a $>$ grabio y molestia e injust $<$ ici $>$ a. $\{25\}$ Juro a esta $\dagger$. Dios es testigo desta verdad. Digo esto por alcan-\{26\} zar just $<$ ici $>$ a, sin malicia ninguna [rúbrica] $\{27\}$ Clara Ve mujer de $\{28\}$ Diego Cen.\{29\}//

\section{Análisis del documento}

En cuanto al nivel gráfico-fonológico, en el documento se registran fenómenos vocálicos como las vacilaciones: 'veniendo', 'confisión'; el empleo de " $y$ " con valor vocálico en posición inicial, interior y final de palabra, lo cual es muy frecuente en los escritos medievales y novohispanos: 'yglesi', 'yndio,' 'traygo,' 'oydos' y 'fuy'.

Entre los consonánticos las epéntesis: 'escharos', y casos de apócope: 'volunta', confies o de sílabas completas, aplología, como 'entien' (entiendo), 'lo prime' (lo primero). Asimismo, se identifica un caso de consonantismo: 'sacristiya'. También común a esta época, y a la anterior, el uso de "u" con valor consonántico, como en 'gouernador', 'auer,' 'absoluer', es decir, este fenómeno se registra en otros documen- 
tos novohispanos y españoles tanto del siglo XVI como del XVII. Además, se documenta la vacilación en el uso de las consonantes "v" y "b" entre el vocablo 'buestro' y la abreviatura 'vro'. Lo interesante aquí es que cuando se refiere a la abreviatura reconoce la forma que debe ser, pero cuando se desata utiliza 'b', quizás se deba a que sólo identifica cómo se escribe la palabra abreviada. Se documenta el fenómeno de lambdacismo, el uso de "l” por "r", en 'y les pondí. En cuanto al grupo 'nm', en una misma palabra, o en diferentes, hay reducción y variación en la escritura, ya que escribe el vocablo como tal y en otros casos lo separa: 'comigo', 'con migo' y 'comi suegro'. Otro fenómeno que se encuentra es el uso de "s" en lugar de " $z$ " en la palabra 'vos' (voz), lo cual dada la fecha del documento indica la presencia de seseo.

Finalmente, los siguientes dos fenómenos nos permiten, por un lado, señalar un indicio de aspiración: 'En mi confision declarare de pie a cabeza' (de pies a cabeza); por el otro, la presencia: 'jacristiya' (sacristía).

En el nivel sintáctico los fenómenos son los siguientes: se aprecia la omisión del verbo 'ser' en la construcción: 'testimonio falso que me levantan por mi suegro Francisco Cen' (testimonio falso que me levantan por ser mi suegro Francisco Cen), este fenómeno se debe a un proceso neto $\mathrm{y}$ frecuente de contacto con la lengua maya (como un calco de la construcción del maya yucateco en el que se involucra la palabra "tumen o men". Así como la falta de la preposición a: 'que no vengo eso' (que no vengo a eso). También se identifica el empleo de algunas preposiciones diferentes del uso actual, por ejemplo: 'respondí que en ninguna manera' o 'cacique y gobernador en el pueblo'. Resulta interesante el 
empleo del 'se' reflexivo 'recuérdose' para referirse a una acción pasada: 'lo prime que hizo conmigo requerdose dixo que yo'. Además, el uso de reiteraciones, por ejemplo: 'entra en la sacritiya con él a hazer pecado con él', fenómeno que tiene su explicación en la influencia del maya. Asimismo, se detecta la falta de concordancia en las siguientes oraciones: 'En la cuaresma pasado fui a confesarme con él', 'enbia buestro marida a llamar', 'estos y otras mucha cos me dixo', 'y si no vas vuestro marido y tu suegro haré por donde escharos deste pueblo'. Los casos de la falta de concordancia no son fortuitos, se deben a la interferencia de la lengua indígena en la que no hay marcas morfológicas ni léxicas de concordancia. En otros casos los elementos utilizados no siguen la norma del español: 'y por esto entien<do $>$ que tiene odio comigo' (y por esto entiendo que me tiene odio), o en: 'haré por donde escharos deste pueblo y quitarle su cacicazgo' (haré por echaros deste pueblo), o también en 'y entonces cumpliré voluntad? (y entonces cumpliré mi voluntad'?). También se registra la omisión del pronombre de OD: 'en ninguna manera podía hazer (lo) en presencia de jentes', o en: 'todo lo que me diera todo os (lo) dará, 'buscaré cómo enviar(lo) fuera'. Además de incorporar el artículo indefinido en una oración que no hace falta: 'es por un odio que tiene el padre Diego'. Se documenta la falta de conector en: 'recuérdose dixo' (recuérdose que dixo) y en 'vuestro marido es un pobre indio no tiene de qué sustentarte' ('es un pobre indio que no tiene'), los ejemplos anteriores se justifican porque en la lengua maya no es necesario el uso de conectores, la yuxtaposición es un recurso para conectar oraciones subordinadas. En otra instancia, se utiliza indicativo 
de presente en lugar del subjuntivo 'dixo que yo entra' ('dixo que yo entrara'). Estos casos se deben al deficiente empleo del español de la escribiente. El documento contiene verbos conjugados en la persona que no corresponde: 'que todo lo que me diera todo os dará (daré)'.

En cuanto al nivel léxico y semántico se documentan fenómenos interesantes, ¿en qué radica su importancia? En que dan cuenta de las transformaciones que sufrieron estas voces indígenas. Por ejemplo el empleo de topónimos mayas: 'Yaxcucul'. Con base en Pacheco Cruz significa "lugar donde se adora primero a dios", ya que proviene de los vocablos Yax, primer, o; kú, dios, nido y kul, contracción de kuul, que quiere decir venerar, adorar y, por tanto, se debe escribir: 'Yaxkúkuul'. ${ }^{1}$ Asimismo, dos antropónimos mayas: Cen y Ve. En ellos se aprecia también la alteración que sufrieron estas voces al ser adaptadas al español, "ya que es lógico pensar que [...] no podían identificar todas las voces mayas y sólo advertirían las más parecidas a su sistema o las más diferentes, sin contar con un sistema de escritura para graficarlas" (Mora Peralta, 2008: 48-49). Con base en lo anterior podemos decir que no registramos el apelativo Cen, lo más cercano es Ceh que quiere decir 'venado'. Sin embargo, nos

\footnotetext{
${ }^{1}$ Santiago Pacheco Cruz (1953: 250-251) apoyándose del Diccionario de Motul define esta voz como "lugar de la primera adoración" que viene de Yax lo dicho i Kukuul, adoración etc., o igualmente podría interpretarse como: "lugar de la cosa de color verde", que esto da a entender la palabra pronunciada en dos partes, YAX KUKUL, como lo trae el Diccionario Motul en la página 451. En relación con la voz KUUL, el mismo Diccionario dice en la página 527 lo que sigue: “kuul: cosa divina”, i la "Coordinación etc., en la página 43, dice: "Kuul, tah Adorar, tener por Dios” i por último, el doctor Solís Alcalá, en la página 16 de su libro, dice: “Adorar. v. tr. Kultal”. pp. 250-251.
} 
apegamos a que el apelativo correcto debió ser Ceh, debido a que su uso es bastante común en el territorio peninsular. Asimismo, consideramos que el patronímico Ve corrió con la misma suerte y, por tanto, debe ser Bee, Beej o Beel escrito con la consonante oclusiva bilabial sonora ' $b$ ' y vocal doble 'e', y que tampoco lograron identificar la consonante glotal fricativa en posición final 'j, o bien la consonante lateral en final de palabra 'l', y cuyo significado es 'camino'. ${ }^{2}$

También documentamos la voz 'guapil' (huipil del náhuatl huipilli), y un vocablo taíno: 'naguas' (f. saya interior de tela blanca.)

Así pues, para el nivel semántico, las palabras 'fatigando' y 'conceder', que si bien no poseen un uso frecuente, son voces que continúan en el léxico del español de México con el mismo significado. Con respecto a la primera, Covarrubias la define como: 'fatigarse, congoxarse, darse mucha priessa en hacer una cosa, hasta cansarse en demasía" (1995: 398). La segunda, no la documenta. Por nuestra parte consideramos que esta voz, 'conceder', no se utilizaría en este contexto.

Finalmente, y con los datos expuestos líneas arriba podemos decir que la mayoría, o muchos de los fenómenos descritos, a través de la carta de Clara Ve, se deben claramente a la interferencia de la lengua materna, en este caso el maya.

Resulta de gran trascendencia analizar este tipo de documentos escritos en español por manos indígenas, ya que

\footnotetext{
${ }^{2}$ Mora Peralta, 2008: 52 y 60. Asimismo, debemos decir que Bracamonte y Sosa identifica a estos apelativos, escritos de la siguiente forma: Ceh y Be (2007: 29).
} 
son una pieza cardinal para los estudios del español colonial que ha sido escasamente tratado, y que dan pauta para explicar muchos fenómenos que se presentan actualmente en el español de la zona maya, en eso estriba su invaluable valor.

\section{Bibliografía}

Arias Álvarez, Beatriz (2014), Documentos públicos y privados del siglo XVI. Textos para la historia del espanol colonial mexicano 1, México, Universidad Nacional Autónoma de México.

_ (1997), El español de México en el siglo XVI. Estudio filológico de quince documentos, México, Universidad Nacional Autónoma de México.

Bastarrachea Manzano, J. Ramón, Ermilo Yah Pech y Fidencio Briceño Chel (1992), Diccionario básico español- maya- español, Mérida, Editores Maldonado. Bastarrachea Manzano, J. Ramón y J. Manuel Canto Rosado (2003), Diccionario maya popular, México, Instituto de Cultura de Yucatán.

COREECOM, http://www.iifl.unam.mx/coreecom/

Covarrubias Orozco, Sebastián de (1995), Tesoro de la lengua castellana o española, Madrid, Castalia.

Mora Peralta, Idanely (2008), Topónimos y antropónimos mayas en documentos coloniales del siglo XVII, tesis de licenciatura, México, Universidad Nacional Autónoma de México. 
Pacheco Cruz, Santiago (1953), Diccionario de etimologías toponímicas mayas, Mérida, Oriente.

Real Academia Española, Diccionario de la lengua española, http://www.rae.es/recursos/diccionarios/drae [consultado: 17/07/2015] 
